\title{
Adult Otogenic Meningitis in the Pneumococcal Conjugated Vaccines Era
}

\author{
Tal Marom ${ }^{1}$ Shay Shemesh ${ }^{1} \quad$ Nadeem Habashi $^{1} \quad$ Ofer Gluck $^{1}$ Sharon Ovnat Tamir ${ }^{1}$ \\ 1 Department of Otolaryngology-Head and Neck Surgery, Samson \\ Assuta Ashdod University Hospital, Ben-Gurion University of the \\ Negev Faculty of Health Sciences, Ashdod, Israel \\ Int Arch Otorhinolaryngol 2020;24:e175-e181.

\begin{abstract}
Address for correspondence Tal Marom, MD, Department of Otolaryngology-Head and Neck Surgery, Samson Assuta Ashdod University Hospital, Ben Gurion University of the Negev Faculty of Health Sciences, 77641 Ashdod, Israel

(e-mail: talmarom73@gmail.com).
\end{abstract}

\begin{abstract}
Introduction Streptococcus pneumoniae is a major pathogen of otogenic meningitis (OgM), the most commonly reported intra-cranial complication of otitis media (OM). Objectives To study the changes in adult OgM patients in the pneumococcal conjugated vaccines (PCVs) era.

Methods Retrospective cohort of adults presenting with concurrent OM and meningitis in a secondary medical care center between 2005 and 2015. Data collected included demographics, OM-related symptoms, cerebrospinal fluid (CSF) and ear culture results, pre- and during hospitalization antibiotic treatment, imaging findings, and complications. We compared the pre-PCV years (2005-2009) with the post-PCV years (2010-2015). Outcomes were 1) incidence of all-cause adult OgM from the total meningitis cases; 2) impact of PCVs on the clinical presentation of OgM and bacteriology.

Results Otogenic meningitis was diagnosed in 26 out of $45(58 \%)$ cases of all-cause meningitis admissions. Of those, $10(38 \%)$ were male, with a mean age of 62.4 years old. Ear-related signs and symptoms were documented in $70 \%$ of the patients, and OgM was diagnosed following imaging studies in 6 out of $26(23 \%)$ patients. All of the patients received intravenous antibiotic therapy, and 7 (27\%) patients required surgical

Keywords

- otitis media

- meningitis

- adult

- imaging

- computed tomography interventions: 6 required myringotomy and 1 required mastoidectomy. There were 12 (46\%) patients in the pre-PCV years and $14(56 \%)$ in the post-PCV years. The positive pneumococcal CSF and ear culture rates remained high and unchanged $(\sim 75 \%)$. There were no significant changes in the clinical presentation or mortality rates.

Conclusion Micro-otoscopy should be included in the routine work-up of any suspected adult meningitis, because OgM is underdiagnosed. Unlike their impact on pediatric otitis media, PCVs did not change the epidemiology and bacteriology of OgM.
\end{abstract}

\section{Introduction}

Intracranial complications secondary to otitis media (OM) arise when the infectious/inflammatory process extends beyond the temporal bone to the adjacent organs, such as the dura. With the advent of antibiotic and surgical therapies over the last century, the incidence of intratemporal/intracranial complica- tions from OM has substantially decreased. ${ }^{1,2}$ Otogenic meningitis (OgM) is the most commonly reported intracranial complication of $\mathrm{OM}$ in adults, and its incidence rate has been reported to range from 0.32 to $0.42 \%$ of all adult OM cases. ${ }^{3-7}$ Otogenic meningitis is typically diagnosed in adults presenting with a recent history of OM, with signs and symptoms suggestive of meningitis. After obtaining a cerebrospinal fluid (CSF) received

November 9, 2018

accepted

August 11, 2019
DOI https://doi.org/

10.1055/s-0039-1697995. ISSN $1809-9777$.
Copyright (c) 2020 by Thieme Revinter

Publicações Ltda, Rio de Janeiro, Brazil
License terms

(요 (1) $\Theta \circledast$ 
sample for chemistry and bacteriology via lumbar puncture (LP), the mainstay of OgM treatment consists of parenteral antibiotics and corticosteroids. Myringotomy can also be of a therapeutic value, because it aims to drain the middle ear cavity and obtain cultures. This is in contrast with the common practice in the preantibiotic era, which included cortical/radical mastoidectomy. ${ }^{8}$

Unlike the pediatric population, who had seen a major intervention aimed to decrease the burden of pneumococcal infections with the implementation of pneumococcal conjugate vaccines (PCVs), adult patients, who are not the usual target population for these vaccines, may benefit from the herd immunity effect. A recent study from Connecticut, USA, evaluated the proportion rates of invasive pneumococcal disease (IPD) cases, including meningitis, among adults aged $>40$ years old. The study showed that in areas with a higher proportion of children that did not complete the recommended 4-dose PCV7 series, there was a higher incidence of overall adult IPD cases, including meningitis, caused by PCV7 serotypes. ${ }^{9}$ A contemporary Dutch audit also showed that following the sequential PCV7 and PCV10 immunization of the pediatric population in the Netherlands, the overall IPD incidence proportionally decreased also in men aged between 20 and 39 years old, who, likewise, were not the target population of these vaccines. ${ }^{10}$ Yet, the data on the changing epidemiology of adult $\mathrm{OgM}$ is partial, since most studies pool meningitis cases with other IPDs, or do not differentiate OgM from all-cause meningitis cases, and conclusions are hard to withdraw. In this single-center report, we sought to report on our experience in a subgroup of adult patients with OgM.

\section{Patients and Methods}

\section{Ethical Considerations}

The present study was approved by the local Institutional Review Board (protocol number: WOMC-0229-16).

\section{Objectives}

The primary objective was to study the incidence of OgM from all-cause adult meningitis cases, and the secondary outcome was to examine the impact of the indirect effect of the implementation of PCVs in the local National Immunization Program (NIP) on adult OgM cases.

\section{Study Design and Population}

We identified retrospectively all adult patients ( $>18$ years old) who presented to the emergency department and were later hospitalized between January 1, 2005 and December 31, 2014 with acute meningitis and had International Classification of Diseases-9 (ICD-9) discharge codes of acute meningitis (320.X, bacterial meningitis; 321.X, meningitis due to other organisms; and 322.X, meningitis of unspecified cause). Adults with previous ear/mastoid disease (i.e., cholesteatoma) or surgery were excluded, as well as patients who developed acute meningitis secondary to neurosurgical procedures or if they had any known immunodeficiencies.

\section{Setting}

The study was performed in a secondary healthcare center located in central Israel. This hospital is one of the 9 public hospitals in the greater Tel Aviv area, which provide healthcare to 3.85 million people (2016 data), and patients have access to all of them when needed. Our local community has some 300,000 adults, and there were no appreciable differences in the population size during the study years. On-call neurology and otolaryngology consultations are available 24/7. Computerized tomography (CT) scans are available 24/ 7, and magnetic resonance imaging (MRI) studies are performed within several hours.

\section{Otogenic Meningitis}

The diagnosis of meningitis was based on clinical findings (nuchal rigidity, photophobia, papilledema, nystagmus, gait instability), in the context of systemic signs and symptoms (fever, headache, nausea/vomiting, confusion, seizures). Laboratory tests for CSF samples were compatible with meningitis findings: elevated opening pressure $\left(>25 \mathrm{~cm} \mathrm{H}_{2} \mathrm{O}\right)$; elevated white blood cell (WBC) count, $>100$ cells $/ \mu \mathrm{L}$; low glucose level $(<40 \%$ of serum glucose) and elevated protein level $(>50 \mathrm{mg} / \mathrm{dL})$. All CSF samples were also sent for Gram staining and standard microbiological cultures.

Per our protocol, any patient with suspected meningitis undergoes neurologic and ophthalmologic evaluation, and is always sent for a head computed tomography (CT) before LP is performed. Routine otolaryngologic evaluation for each adult meningitis is not performed.

The diagnosis of $\mathrm{OgM}$ was made in patients presenting with concurrent meningitis and OM, diagnosed on otoscopy and/or ear swab culture, but also ad hoc, after reviewing imaging studies, mostly CT scans, which had shown the presence of opacification in the tympanomastoid cavity. In these ad hoc cases, patients were subsequently referred for otomicroscopy in the otolaryngology service, which reinforced the diagnosis of OM and were managed accordingly.

Medical charts of eligible patients were reviewed and verified for the accuracy of OgM episodes independently by 2 authors (Shemesh S., Tamir S. O.). Data collected and analyzed included age, gender, history of current disease, history of previous OM episodes, past medical and surgical history, laterality, pre-hospital antibiotic treatment, OgM symptoms and signs, ear and CSF culture results, antibiotic treatment during hospitalization, timing of imaging studies, findings on CT/MRI studies, OgM-related sequelae surgical interventions during hospitalization (myringotomy, mastoidectomy or neurosurgical drainage). We did not collect data on viral studies because they were not consistently performed in all adult OgM cases.

Because meningitis is an IPD, it is requested by the Israeli Ministry of Health to send all Streptococcus pneumoniae isolates from CSF cultures for serotyping in its central laboratory for national surveillance purposes. These data are not sent to the sending hospitals because it has no additive, practical therapeutic value in the management of meningitis patients in real time. Because this information is irrelevant for decision-making purposes (unlike bacterial antibiotic sensitivity tests), we did not collect this data. 


\section{Pneumococcal Conjugated Vaccines Periods}

In brief, PCV7 was introduced in the Israeli NIP in children in July 2009, which was shortly replaced later in November 2010 by PCV13, which offered a broader coverage of 6 additional serotypes. Pneumococcal conjugated vaccines are routinely administered to infants only in their first 2 years of life. By June 2011 and December 2012, 80\% and 90\%, respectively, of Israeli children between 7 and 11 months old received $\geq 2$ PCV7 and/or PCV13 doses; by June 2014, 95\% received $\geq 2$ PCV13 doses. By June 2011 and December 2012, 36 and $87 \%$, respectively, of children between 24 and 35 months old, received $\geq 3$ PCV7/PCV13 doses; by June 2014, 91\% received $\geq 3$ PCV13 doses. ${ }^{11}$

The period between 2005 and 2009 was considered as the "pre-PCV years", when the general population could be considered as naïve, vaccine-free, and the period between 2010 and 2014 was considered as the "post-PCV years", when the pediatric population has been gradually vaccinated. Adults are not requested to be immunized with PCVs, but may indirectly be affected by this intervention in a nonmeasurable way. Therefore, we hypothesized that the clinical presentation of OgM may have changed in the post-PCV years.

\section{Statistical Analysis}

Data were recorded on a Microsoft Excel spreadsheet (Microsoft Corporation, Redmond, WA, USA). The unit of analysis was an OgM episode. Statistical analysis was performed using IBM SPSS Statistics for Windows, Version 21.0 (IBM Corp., Armonk, NY, USA). Events were compared using the chi-squared test or the Fisher exact text, appropriately. All of the tests were two-sided and were considered significant at $p<0.05$.

\section{Results}

\section{Demographics}

Overall, there were 45 cases of all-cause adult meningitis. Of them, $26(58 \%)$ cases were classified as OgM, and were the focus population of our study. Of them, 20 were diagnosed by concurrent otomicroscopy and diagnostic imaging modality (CT scan) upon admission, and 6 additional cases were initially diagnosed as having normal otoscopy or did not undergo otoscopic examination before the CT scan had been performed. A total of 10 (38\%) of the study patients were male. None of the patients had received any PCV dose. Demographic and clinical data are presented in -Table 1. In $3(12 \%)$ cases, a history of recent $\mathrm{OM}(<14$ days prior to admission) was documented. A preadmission antibiotic treatment was documented in $5(19 \%)$ patients. The mean hospitalization duration was 20 days (range: 3-81 days).

\section{Otogenic Meningitis Presentation and Management}

Upon admission, at least 2 typical signs/symptoms of meningitis were documented in 25 (96\%) patients, and included headache (23 patients; $88 \%$ ), nuchal rigidity $(n=23 ; 88 \%$ ), body temperature $>38.1^{\circ} \mathrm{C}(n=21 ; 81 \%)$, confusion $(n=21$; $81 \%)$, nausea or vomiting $(n=19 ; 73 \%)$, photophobia $(n=7$; $27 \%$ ) and papilledema ( $n=2 ; 7 \%)$. Otogenic signs/symptoms
Table 1 Demographic and clinical presentation of otogenic meningitis $(n=26)$

\begin{tabular}{|l|l|}
\hline Variance & Value \\
\hline Male & $10(38 \%)$ \\
\hline Mean age, years old (SD) & $62.4(13)$ \\
\hline Previous otologic history & $3(11 \%)$ \\
\hline Preadmission antibiotic treatment & $5(19 \%)$ \\
\hline Otitis media (on otoscopy) & $21(81 \%)$ \\
\hline Otogenic meningitis diagnosed after CT & $6(23 \%)$ \\
\hline Myringotomy performed & $6(23 \%)$ \\
\hline
\end{tabular}

Abbreviations: CT, computed tomography; SD, standard deviation.

were not universal: 18 (69\%) patients reported otalgia, and 5 (19\%) reported otorrhea prior to hospital admission.

Intravenous antibiotic treatment was administered in all OgM cases. Ceftriaxone was the most frequent treatment, prescribed in 24 (92\%) patients, followed by vancomycin $(n=14 ; 54 \%)$ and ampicillin $(n=11 ; 42 \%)$. In $18(69 \%)$ patients, the antibiotic treatment was switched during the hospitalization course, due to bacterial culture results and antibiotic resistance studies reported during the course of the hospitalization.

\section{Bacteriology}

Cultures were collected and sent for bacterial studies from LP samples (24 patients, 92\%), myringotomy ( $n=18 ; 69 \%$ ), spontaneous otorrhea $(n=1 ; 3 \%)$ and from the mastoid cavity during surgery $(n=1 ; 3 \%)$. Of the 28 analyzed cultures, 22 (79\%) grew bacteria. Of the positive cultures, S. pneumoniae grew in $20(91 \%)$ cases, Listeria monocytogenes in $1(5 \%)$ case, and Haemophilus influenzae in $1(5 \%)$ case. There were no positive cultures for other typical meningitis/OM pathogens, such as Neisseria meningiditis or Moraxella catarrhalis. There were no cases of mixed bacterial growth. One patient presented with 2 different OgM episodes, with a complete resolution between them. His $1^{\text {st }} \mathrm{CSF}$ culture grew S. pneumoniae, and the $2^{\text {nd }}$ and $3^{\text {rd }}$ cultures obtained during the subsequent hospitalization 3 months later also grew $S$. pneumoniae again in both CSF and mastoidectomy cultures, but had different sensitivities to antibiotic agents from the S. pneumoniae isolated in the $1^{\text {st }}$ episode. He had no OM episodes in between these episodes.

All of the the 26 (100\%) OgM patients had CSF cultures obtained during LP. Of them, 20 (77\%) had also ear cultures, obtained during myringotomy, swabbing of the external ear and mastoidectomy. Of them, 8 (40\%) had bacterial growth. In these patients, ear cultures were obtained via myringotomy in 6 (75\%) patients, on the $2^{\text {nd }}$ day following admission, on average (range 0-8 days); culture from spontaneous otorrhea in $1(13 \%)$ patient, and culture from mastoidectomy cavity in $1(13 \%)$ patient. - Table 2 shows the concordance between the CSF and the ear cultures in those patients. Of the 6 patients who underwent myringotomy, 3 (50\%) patients had identical CSF and ear culture results (which both grew S. pneumoniae). The other $3(50 \%)$ patients who underwent 
Table 2 Comparison between cerebrospinal fluid and middle ear cultures $(n=8)$

\begin{tabular}{|l|l|l|l|l|}
\hline Patient & Ear Culture Source & Day of Culture & CSF Culture Result & Ear Culture Result \\
\hline 1 & Myringotomy & 1 & Streptococcus pneumoniae & Streptococcus pneumoniae \\
\hline 2 & Myringotomy & 0 & Streptococcus pneumoniae & Staphylococcus coagulase negative \\
\hline 3 & Myringotomy & 0 & Streptococcus pneumoniae & No growth \\
\hline 4 & Myringotomy & 3 & No Growth & Staphylococcus coagulase negative \\
\hline 5 & Myringotomy & 8 & Streptococcus pneumoniae & No growth \\
\hline 6 & Spontaneous otorrhea & 1 & Streptococcus pneumoniae & Streptococcus pneumoniae \\
\hline 7 & Mastoidectomy & 3 months & Streptococcus pneumoniae & Streptococcus pneumoniae \\
\hline 8 & Myringotomy & 1 & Streptococcus pneumoniae & No growth \\
\hline
\end{tabular}

myringotomy had Staphylococcus coagulase negative (which was interpreted as contaminant) or no growth in their ear cultures. Because the patients had already been treated with a broad-spectrum antibiotic treatment that covered these pathogens beforehand, there was no need for a change in the antibiotic policy.

During the pre-PCV years, there were 12 (46\%) cases, compared with 14 (54\%) cases during the post-PCV years. Since there were no appreciable changes in the population size in our area, this reflects a stable OgM admission rate during the whole study period. Of the 12 pre-PCV cases, 9 (75\%) had S. pneumoniae grown in their CSF cultures, while of the 14 post-PCV cases, 11 (78\%) were positive for $S$. pneumoniae in their CSF cultures $(p=0.59)$. Since OM is not considered to be IPD and serotyping from ear cultures is not routinely performed in clinical settings like ours, we could not show if there was a serotype shift phenomenon from vaccine to non-vaccine strains.

The average age and hospitalization duration was similar in both groups. There were significantly more patients with a medical history of diabetes mellitus and hypertension in the post-PCV years (25\%, pre-PCV years versus $71 \%$, post-PCV years). A total of $5(36 \%)$ patients in the post-PCV years had a bilateral disease, while none of the pre-PCV patients had a bilateral disease. Due to these small numbers, we could not perform the statistical analysis.

Complications varied from neurological sequelae, such as partial to complete paresis and alteration in consciousness level, permanent sensoneural hearing loss, to cardiac arrest and death. In the pre-PCV years, there were $4(33 \%)$ patients with OgM sequelae, compared with $6(43 \%)$ in the post-PCV patients $(p=0.39)$. The overall mortality rate was $15 \%: 3$ (25\%) patients in the pre-PCV group eventually died from OgM complications, and 1 (7\%) patient in the post-PCV died of neurological sequalae $(p=0.12)$.

\section{Imaging Studies}

All of the patients underwent CT scans, and 2 patients also underwent MRI studies for suspected intracranial complications (both were in the post-PCV years). A total of 4 patients (15\%) had impacted cerumen blocking the external ear canal, plausibly leading to the delayed diagnosis of OgM (-Fig. 1A).
As for findings in imaging studies, 6 (50\%) patients in the pre-PCV years had tympanomastoid opacifications alone, while the other patients had concomitant rhinosinusitis findings (-Fig. 1B). Osteomyelitis of the temporal bone was observed in $6(43 \%)$ patients. Tegmen dehiscence was the most common pathology ( - Fig. 1C). In the post-PCV years, there were 7 (50\%) patients with isolated OgM, while the others had concomitant rhinosinusitis findings in their imaging studies. Osteomyelitis was observed in $5(42 \%)$ patients, with, again, tegmen dehiscence as the common pathology, and one case of petrous apicitis ( - Fig. 1D). We did not find any comorbidity that had significant correlation with either pathology observed in imaging studies.

\section{Discussion}

We report that $58 \%$ of all-cause adult meningitis in our cohort could be classified as originating from an otogenic source. The overall outcome of adult OgM patients treated with intravenous antibiotic therapy was good, and only a few required surgical interventions, which were minor in most cases (only myringotomy). There were no considerable differences in the pre- and post-PCV years in the epidemiology and clinical presentation of adult $\mathrm{OgM}$. The present retrospective study did not find any change in the proportion of OgM growing S. penumoniae on ear or CSF cultures.

It is widely accepted that $\mathrm{OM}$ is the most common cause for pediatric meningitis, due to the proximity of the tympanomastoid cavity to the dura, as well as the presence of inner ear malformations and the relative ease for the hematogenous spread of the infectious process from the middle ear cleft. ${ }^{12}$ Connections between the hematopoietic bone marrow of the temporal bone and the middle ear in autopsies of infants with meningitis and OM have also been suggested as a potential route for the infection. ${ }^{13}$ However, OM as a cause for adult OgM is a less accepted notion, and in our view, under-appreciated. Ear-related signs and symptoms were noticeable in only $70 \%$ of our patients (a few patient families were interviewed and stated this fact only after a "cause directed" interview, because the patient could not be interviewed due to his neurological status), and $\mathrm{OM}$ as a cause for meningitis was absent in $20 \%$ of the cases. We showed an incidence rate of $56 \%$ of $\mathrm{OgM}$ from all-cause meningitis, 

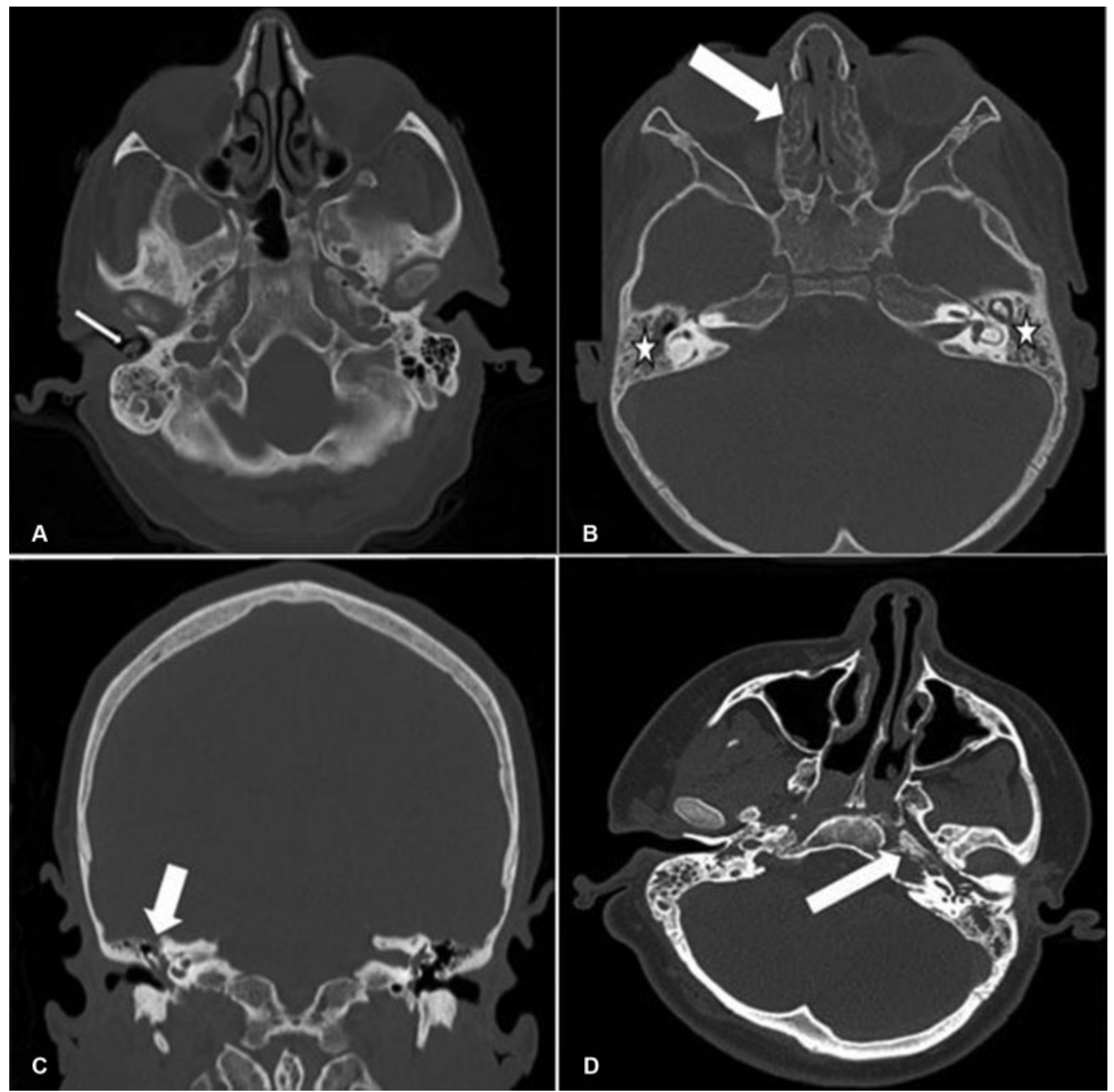

Fig. 1 Representative computed tomography (CT) Scans of Adult Patients with Ad hoc Otogenic Meningitis. (A) Axial CT scan showing an impacted cerumen (white arrow), obscuring the right tympanic membrane. (B) Axial CT scan showing bilateral opacifications of the tympanomastoid cavities (white star) as well as the middle ear cleft and ethmoidal sinusitis (white arrow). (C) Coronal CT scan showing opacification of the right middle ear cavity and ipsilateral dehiscence of the tegmen tympani (white arrow). (D) Axial CT scan showing opacification of the left petrous apex of the temporal bone (white arrow), compatible with petrous apicitis.

which is higher than previously published pre-PCV era reports: $15 \%$ (13/87) in Bristol, United Kingdom, ${ }^{7}$ 4\% (8/ 201 ) in Oporto, Portugal, ${ }^{14}$ or $30 \%$ in the Danish National Registry. ${ }^{15}$ Unlike these studies, which also included infants and children, our population consisted of verbal adult patients who reported their symptoms and could be questioned for their complaints.

The association between PCVs and the decrease in pediatric meningitis cases has been previously described in many countries that implemented PCVs in their NIP..$^{16-18}$ In the U. S., the decrease in pneumococcal meningitis was the greatest in the early post-PCV7 period (introduced in 2000), with an additional small decrease in the post-PCV13 years (introduced in 2010). ${ }^{2}$ The most pronounced decline in meningitis admission prevalence occurred in children $<1$ year old, followed by children aged between 1 and 2 years old. In older children, the decrease was modest, in accordance with the acute OM prevalence distribution in the pediatric population.

The introduction of PVCs has positively affected the incidence of IPDs in adults as well, including all-cause meningitis, as observed in the changes in S. pneumoniae serotypes before and after the implementation of PCVs, ${ }^{19}$ in concordance with the observed decrease with the national uptake of the vaccines ${ }^{20}$ and the reduction in overall all-cause and prevalence of 
pneumococcal meningitis. ${ }^{11}$ The biggest evidence comes from an epidemiological study that demonstrated a downward trend in the pneumococcal meningitis hospitalization rates across the U.S. in the post-PCV13 years, but without a substantial change in the fatality rate. ${ }^{21}$ Although this report does not specifically address $\mathrm{OgM}$ as a subgroup, the authors showed that in children $<2$ years old, who are the target population of PCVs and are at the peak age for OM, the average annual pneumococcal meningitis admission rate decreased by $45 \%$, from 2.19 in the late-PCV7 years to 1.20 in the post-PCV13 years $(p=0.10)$. In adults aged between 18 and 39 years old, there was also a significant downward trend, from 0.25 to $0.15(p=0.02)$, similarly to adults aged between 40 and 64 years old, from 0.95 to $0.54(p=0.03)$; and in patients $\geq 65$ years old, the admission rate decreased by $38 \%$, from 1.02 to 0.63 , though it did not reach statistical significance $(p=0.08)$ (all rates shown are for pneumococcal meningitis admission cases per 100,000 individuals). The same trend was also observed in nonpneumococcal meningitis admission rates, but did not reach statistical insignificance. The same decrease has also been reported in Colombia, where the observed over the expected monthly meningitis rate decreased in 90.5\% in the post-PCV period (2012-2015), when compared with the pre-PCV period. ${ }^{22}$ We could not show such a change in the incidence of OgM in the pre- and post-PCV periods, possibly due to the small sample size. Also, we were unable to show such a decrease in the S. pneumonia-positive cultures in the post-PCV years.

Our work is based on a meticulous chart review of selected OgM patients, regarding their ear disease and imaging studies. To our knowledge, this is the first study focusing on this subgroup of adult meningitis patients in the post-PCV era. We showed that ad hoc diagnosis is important and relevant for the diagnosis of OgM early during the disease, due to favorable outcomes. We acknowledge limitations to our work: 1) small study group, proportional to the size of our single center. This limited us from performing meaningful statistical analyses. Yet, other reports also include only a few dozens of patients $4,7,12,23 ; 2$ ) we do not have data on OgM hospitalization rates in our neighboring hospitals; 3 ) no serotype data; and 4) due to the retrospective nature of the present study, we could not get into the details why otoscopy was not performed in all cases or why the interpretation of the first otoscopy was considered to be normal.

\section{Conclusion}

To conclude, OgM is underdiagnosed. Ear-related signs and symptoms in adult patients with meningitis are not universal, and OM as a cause for meningitis can be easily missed. Since OM pathogens may be different from meningitis pathogens and the need to drain the middle ear in OgM cases, we suggest that micro-otoscopy for any adult OgM should be in the routine work-up for any patient with meningitis. When the reason for presumptive meningitis is unclear, patients should be evaluated by an otolaryngologist in addition to imaging and ophthalmologic evaluation. Once OM has been diagnosed on otoscopy, the otolaryngologist should be involved in the management of these patients.

\section{Compliance with Ethical Standards}

The present research involved human participants. Informed consent has been waived due to the retrospective nature of the study. An institutional review board permission has been granted.

\section{Comment}

The present work has been conducted at the Edith Wolfson Medical Center, where all of the the authors worked.

Conflicts of Interests

The authors have no conflicts of interests to declare.

\section{References}

1 Orji FT, Ukaegbe O, Alex-Okoro J, Ofoegbu VC, Okorafor IJ. The changing epidemiological and complications profile of chronic suppurative otitis media in a developing country after two decades. Eur Arch Otorhinolaryngol 2016;273(09):2461-2466

2 Tawfik KO, Ishman SL, Altaye M, Meinzen-Derr J, Choo DI. Pediatric Acute Otitis Media in the Era of Pneumococcal Vaccination. Otolaryngol Head Neck Surg 2017;156(05):938-945

3 Leskinen K, Jero J. Acute complications of otitis media in adults. Clin Otolaryngol 2005;30(06):511-516

4 Wanna GB, Dharamsi LM, Moss JR, Bennett ML, Thompson RC, Haynes DS. Contemporary management of intracranial complications of otitis media. Otol Neurotol 2010;31(01):111-117

5 Osma U, Cureoglu S, Hosoglu S. The complications of chronic otitis media: report of 93 cases. J Laryngol Otol 2000;114(02):97-100

6 Kangsanarak J, Navacharoen N, Fooanant S, Ruckphaopunt K. Intracranial complications of suppurative otitis media: 13 years' experience. Am J Otol 1995;16(01):104-109

7 Ibrahim SI, Cheang PP, Nunez DA. Incidence of meningitis secondary to suppurative otitis media in adults. J Laryngol Otol 2010;124(11): 1158-1161

8 Singh B. The management of lateral sinus thrombosis. JLaryngol Otol 1993;107(09):803-808

9 Pingali SC, Warren JL, Mead AM, Sharova N, Petit S, Weinberger DM. Association Between Local Pediatric Vaccination Rates and Patterns of Pneumococcal Disease in Adults. J Infect Dis 2016;213 (04):509-515

10 Wagenvoort GH, Sanders EA, Vlaminckx BJ, de Melker HE, van der Ende A, Knol MJ. Sex differences in invasive pneumococcal disease and the impact of pneumococcal conjugate vaccination in the Netherlands, 2004 to 2015. Euro Surveill 2017;22(10):30481

11 Regev-Yochay G, Katzir M, Strahilevitz J, et al; IAIPD group. The herd effects of infant PCV7/PCV13 sequential implementation on adult invasive pneumococcal disease, six years post implementation; a nationwide study in Israel. Vaccine 2017;35(18):2449-2456

12 Van der Poel NA, van Spronsen E, Dietz de Loos DA, Ebbens FA. Early signs and symptoms of intracranial complications of otitis media in pediatric and adult patients: A different presentation? Int J Pediatr Otorhinolaryngol 2017;102:56-60

13 Terao K, Cureoglu S, Schachern PA, et al. Marrow-middle ear connections: a potential cause of otogenic meningitis. Otol Neurotol 2011;32(01):77-80

14 Santos LC, Simões J, Severo M, Vazquez J, Lecour H. Bacterial meningitis in an urban area: etiologic study and prognostic factors. Infection 2007;35(06):406-413

15 Østergaard C, Konradsen HB, Samuelsson S. Clinical presentation and prognostic factors of Streptococcus pneumoniae meningitis according to the focus of infection. BMC Infect Dis 2005;5:93

16 Marom T, Bookstein Peretz S, Schwartz O, Goldfarb A, Oron Y, Tamir SO. Impact of Pneumococcal Conjugate Vaccines on Selected Head and Neck Infections in Hospitalized Israeli Children. Pediatr Infect Dis J 2017;36(03):314-318 
17 Shinjoh M, Yamaguchi Y, Iwata S. Pediatric bacterial meningitis in Japan, 2013-2015 - 3-5 years after the wide use of Haemophilus influenzae type b and Streptococcus pneumoniae conjugated vaccines. JInfect Chemother 2017;23(07): 427-438

18 de Oliveira LH, Camacho LA, Coutinho ES, et al. Impact and Effectiveness of 10 and 13-Valent Pneumococcal Conjugate Vaccines on Hospitalization and Mortality in Children Aged Less than 5 Years in Latin American Countries: A Systematic Review. PLoS One 2016;11(12):e0166736

19 Corcoran M, Vickers I, Mereckiene J, et al. The epidemiology of invasive pneumococcal disease in older adults in the post-PCVera. Has there been a herd effect? Epidemiol Infect 2017;145(11): 2390-2399
20 Tsaban G, Ben-Shimol S. Indirect (herd) protection, following pneumococcal conjugated vaccines introduction: A systematic review of the literature. Vaccine 2017;35(22):2882-2891

21 Jacobs DM, Yung F, Hart E, Nguyen MNH, Shaver A. Trends in pneumococcal meningitis hospitalizations following the introduction of the 13-valent pneumococcal conjugate vaccine in the United States. Vaccine 2017;35(45):6160-6165

22 Caceres DC, Ortega-Barria E, Nieto J, DeAntonio R. Pneumococcal meningitis trends after pneumococcal conjugate vaccine introduction in Colombia: An interrupted time-series analysis. Hum Vaccin Immunother 2018;14(05):1230-1233

23 Slovik Y, Kraus M, Leiberman A, Kaplan DM. Role of surgery in the management of otogenic meningitis. J Laryngol Otol 2007;121 (09):897-901 